Scaling and critical probabilities for cluster size and LA diversity on randomly occupied square lattices

This content has been downloaded from IOPscience. Please scroll down to see the full text.

2000 J. Phys. A: Math. Gen. 332739

(http://iopscience.iop.org/0305-4470/33/14/309)

View the table of contents for this issue, or go to the journal homepage for more

Download details:

IP Address: 146.175.11.111

This content was downloaded on 07/01/2014 at 07:36

Please note that terms and conditions apply. 


\title{
Scaling and critical probabilities for cluster size and LA diversity on randomly occupied square lattices
}

\author{
I J Tsang, I R Tsang, B De Boeck and D Van Dyck \\ VisionLab, Department of Physics, University of Antwerp - RUCA, Groenenborgerlaan 171, \\ Antwerp B-2020, Belgium
}

Received 10 November 1999

\begin{abstract}
Using Monte Carlo simulations, we report the behaviour of the total number of clusters, the cluster size diversity and the lattice animals (LA) diversity on randomly occupied square lattices. The critical probability associated with the maximum of these variables is determined in comparison with the percolation probability $p_{c}$. Our simulations indicate that $p_{c}$ and the critical probability of the maximum cluster size diversity $p_{c}\left(D_{s \max }\right)$, occur at the same point. As indicated in a previous paper (Tsang I R and Tsang I J 1997 J. Phys. A: Math. Gen. 30 L239), the probability for the maximum number of clusters is obtained at a lower value, $p_{c}\left(N_{\max }\right)=0.27 \pm 0.01$. We describe the cluster identification algorithm used to count the different LA and determined the critical probability for the maximum LA diversity, $p_{c}\left(D_{f \max }\right)=0.45 \pm 0.02$. We derive the exponents characterizing the relation between $D_{s \max }, D_{f \max }$, and $N_{\max }$ and several scaling relations between the variables measured, the lattice size, and the probability of occupation $p$. In addition, we show the scaling behaviour of LA diversity versus cluster size diversity for each value of $p$.
\end{abstract}

\section{Introduction}

Lattice animals (LA) are clusters of connected sites or bonds embedded on a $d$-dimensional lattice. LA represent random clusters which can be found in many systems, its configurational statistics has been a subject of importance because of its application in a variety of problems [1]. The statistics and enumeration of $n$-cell LA have been of much interest [2-4] and various papers have been written on LA in connection with percolation [5-8], branched polymer problems [9, 10] and self-organized criticality [11]. Also known as polyominoes [12], it is usual to make a distinction between free and fixed animals. A free LA is not considered different from another if it can be derived by symmetry operations, while in fixed LA they are regarded as different. In this paper, we are interested in the statistics of fixed LA diversity in relation to the probability of occupation, instead of the more usual problem of the LA enumeration.

Diversity is a widely observed phenomenon in the natural world and its concept has been applied to a variety of problems ranging from biology [13] and evolution [14] to selforganization [15], cellular automata [16], and fractals [17]. Diversity of cluster size has been analysed in several non-equilibrium dynamics which generate a distribution of fragments of size (mass) [18-22] and is a well-suited variable to measure the complexity of fragmentation and aggregation dynamics, as shown in [22].

Recently, we have shown that the structural complexity of randomly occupied square lattices can be defined by the diversity of cluster size [23-25]. Diversity can be described by the amount of different kinds of patterns observed in the system. The types of patterns can be 
defined by considering different physical properties such as size or shape. In this paper, we have used both cluster size and LA as definitions of patterns for the diversity measurement. Note that diversity of cluster size is a measurement on the difference of size scale of the system, while in the LA diversity the differences lie in the shapes of the clusters. Therefore, by examining LA diversity, we are analysing the structure of the system on a finer-grained pattern structure.

The statistics of the number of cluster $N(s, p)$, on random and disordered models, have been quite well analysed, specially in connection with percolation problems [8]. However, on this and other models that generate a distribution of cluster, diversity is a measurement not as greatly studied. Diversity of cluster size is particularly appropriate for a quantitative numerical analysis, since it is a quantifiable macroscopic measurement, and is thus a variable worthy of investigation. On the other hand, LA diversity poses a more challenging problem to be treated by numerical analyses. We describe here an algorithm that identifies and counts the different LA which emerge on the lattice by varying the probability of occupation.

In these simulations, we are interested in the cluster size and fixed-site LA diversity generated by randomly occupying a square lattice with probability $p$, similarly to the ideas in [23-25]. We show the behaviour of LA diversity, cluster size diversity and the total number of clusters in relation to the probability of occupation and the size of the system. Critical probabilities associated with $D_{s \max }, D_{f \max }$, and $N_{\max }$ are derived. A non-trivial scaling relation between the maximum diversity of LA and the maximum number of clusters is determined. Furthermore, we report the scaling relation between LA and cluster size diversity. The numerical simulations and finite-size scaling analysis indicates that $p_{c}$ and $p_{c}\left(D_{s \max }\right)$, occur at the same critical probability.

The structure of this paper is as follows. In section 2 we define mathematically the measurements that are analysed in this system. In section 3 we describe the computer simulations and the LA identification algorithm. In section 4 the critical probabilities associated with the variables measured are derived. In section 5 the scaling relations between these variables are analysed. Finally, in section 6, discussions and concluding remarks are presented.

\section{Complexity, diversity and LA}

In recent years, there has been an increased interest in the study and understanding of complex systems. Presently, there are no defined procedures for a quantitative measurement of complexity and a unifying approach or framework on the characteristics of this concept is still lacking. In various situations, complexity is related to systems which exhibit structures with variations. On the other hand, diversity is widespread in nature and it can be measured by the amount of different kinds of patterns exhibited by the system. In [25], we have used diversity of cluster size as a measurement of the structural complexity on randomly occupied lattices. A cluster is defined as a group of occupied sites connected by a nearest-neighbours relationship. In this paper, we are interested in the fixed LA as pattern defined for the diversity measurement, and on how the cluster size and the fixed LA diversity are related to each other. Even though the system studied is one of the simplest model of randomly disordered systems, an analysis on the LA and cluster size diversity can contribute to a better understanding of this model that is closely related to the percolation and phase transition problems.

The variables of interest i.e. $D_{s}$, diversity of cluster size, $D_{f}$, LA diversity, and $N$, the total number of clusters or LA, were measured as functions of both $L$ and $p$. The mathematical 
definition for the cluster size diversity is given by

$$
D_{s}(p)=\left\langle\sum_{s} \Theta[N(s, p)]\right\rangle
$$

for the diversity of fixed LA,

$$
D_{f}(p)=\left\langle\sum_{f} \Theta[N(f, p)]\right\rangle
$$

and for the total number of LA or clusters,

$$
N(p)=\left\langle\sum_{s} N(s, p)\right\rangle .
$$

Here, $N(s, p)$ is the number of clusters of size $s$ and $N(f, p)$ is the number of clusters of the same fixed LA $f$, in a single experiment, for occupation probability $p ; \Theta(x)$ is the Heaviside function defined as $\Theta(x)=1$ if $x>0$, and $\Theta(x)=0$ otherwise, and the average $\langle\ldots\rangle$ is computed over different experiments.

\section{Simulations}

We perform Monte Carlo simulations on square lattices with size varying from $L=$ $60,100,300,600,1000$ and 2000, with averages taken on 6000, 5000, 2000, 500, 300 and 200 experiments, respectively. The lattices were randomly occupied with probability $p$ ranging from 0.05 to 0.95 with steps of 0.01 between $p=0.25$ to 0.3 and $p=0.4$ to 0.65 , and steps of 0.05 on the other regions. We used the well known Hoshen-Kopelman algorithm [26] to identify the clusters of different sizes. However, for the LA, we developed a specific algorithm to count the diversity of LA based on the site neighbour relationships.

\subsection{LA identification algorithm}

Recently, the enhanced Hoshen-Kopelman (EHK) algorithm [27] has been developed. The EHK algorithm is an improvement on the original HK algorithm and it determines information on the cluster size and on other structural properties of the clusters such as the internal perimeter, radius of gyration or the spatial moments. These measurements yield information on the shape structure of the clusters, but do not differentiate the LA. We propose a cluster identification algorithm for LA [28] to solve this problem. The main idea of this algorithm is to code each cluster site according to the number and position of the occupied nearest-neighbour sites. For each cluster a set of codes equivalent to each cell is composed. The distinction between two LA is performed by comparing each code, site by site, in the ordering sequence in which the cluster is scanned.

The structural characterization of each site of the LA is represented by a vector $V$ in which each component indicates a neighbour cell in one of the four directions. Therefore, $V=(n, e, s, w)$ where each letter, respectively, represents the presence or absence of a neighbour cell in the directions north, east, south, and west. In practice, the nearest-neighbour vector can be coded as an integer where the first four bits are used to assign a possible nearestneighbour configuration. As a result, there are 16 possible codes. An order sequence of each cell is associated with the codes. In this case, we used the order in which the clusters are scanned. So, by comparing the sequence of codes one can determine if two LA are distinct or not. To show that this is sufficient to differentiate the LA, let us try to perform a structural change on a LA without altering the order sequence and contents of the nearest-neighbour 


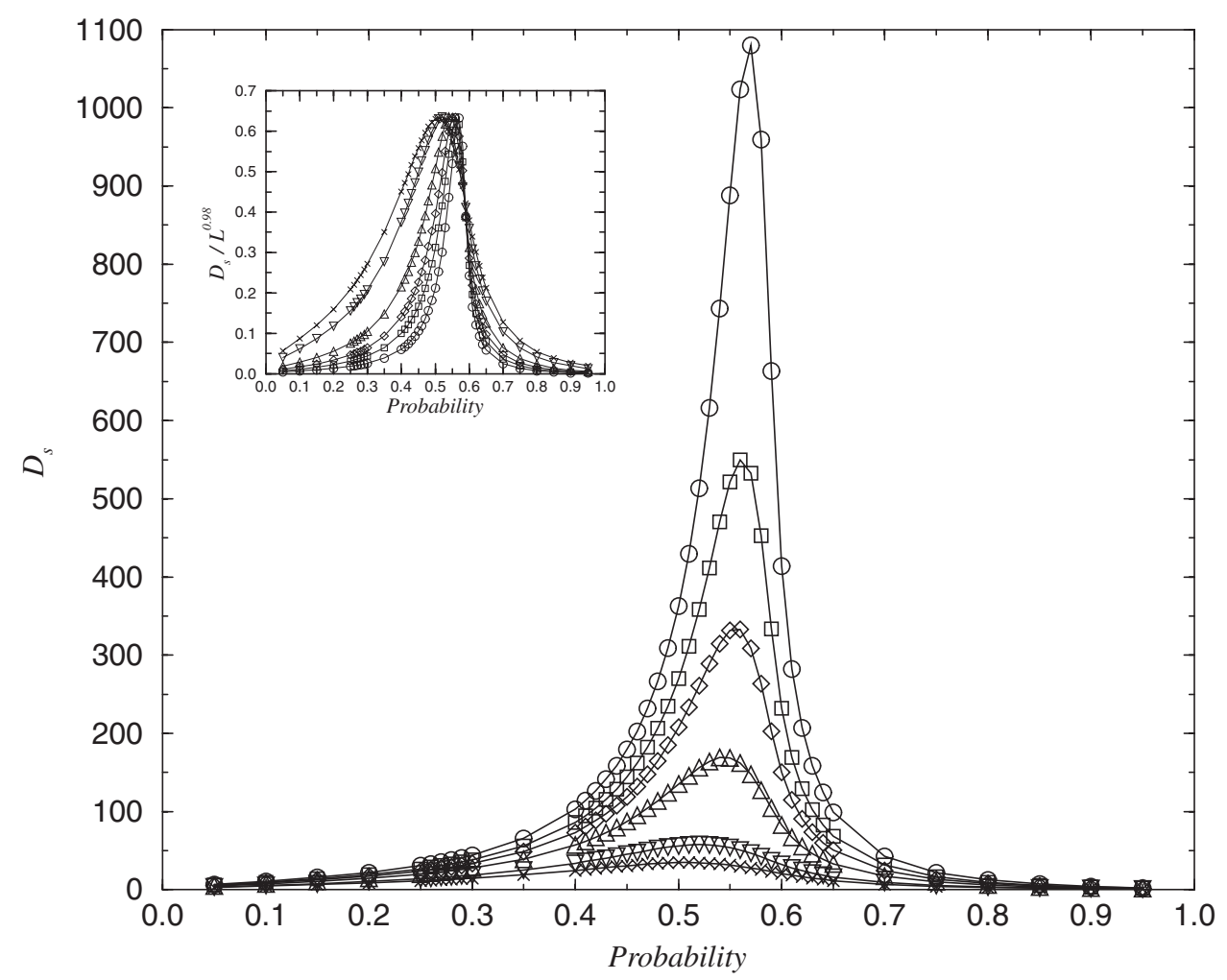

Figure 1. The behaviour of cluster size diversity as a function of the probability of occupation for $L=2000(\bigcirc), 1000(\square), 600(\diamond), 300(\triangle), 100(\nabla), 60(\times)$. The inset shows the ratio $D_{f} / L^{0.98}$ against $p$.

codes. Here, two shapes will be comparable only if they have the same amount of cells. A change in the position of any cell $\mathcal{A}$ will infer a change in its ordering label and/or in its nearest-neighbour code content, moreover it also causes a change in at least another cell $\mathcal{B}$ neighbouring to cell $\mathcal{A}$, since any of the cells must be connected. Consequently, it will also cause a change in at least another nearest-neighbour code and/or in its ordering label.

The computational time complexity for this algorithm is not linear as the EHK algorithm and it also requires a larger amount of memory space, since the sets of nearest-neighbour codes representing the LA need to be stored. For this reasons simulations for a larger number of configuration and lattice sizes are more computational demanding. A more detailed description of this algorithm and its implementation within the enhanced Hoshen-Kopelman algorithm framework are currently under preparation [28].

\section{Critical probability}

On randomly occupied lattices the probability of occupation determines the structure of the system. A low value of $p$ generates a lattice with small and disconnected clusters, while with $p$ near to 1 a single totally connected cluster is generated. However, with an intermediate value of $p$ a configuration with maximum diversity emerges. In this configuration, clusters with all size scales can be found. 


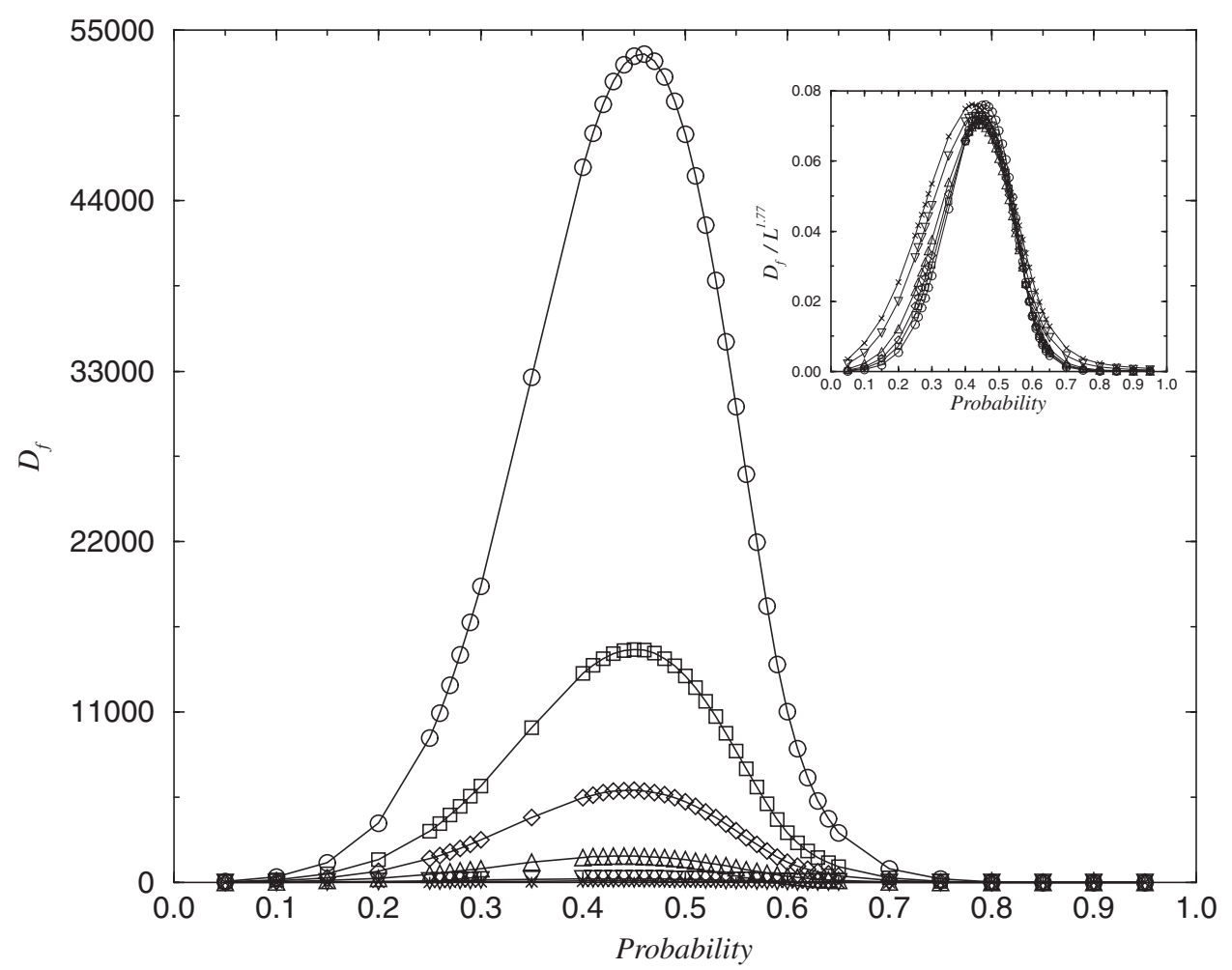

Figure 2. The behaviour of fixed animal lattice diversity as a function of the probability of occupation for $L=2000(\bigcirc), 1000(\square), 600(\diamond), 300(\triangle), 100(\nabla), 60(\times)$. The inset shows the ratio $D_{f} / L^{1.77}$ against $p$; with the normalization factor the curves show a reasonable collapse.

In figures 1 and 2 we show the behaviour of cluster size and fixed LA diversity as a function of the probability of occupation for different values of $L$. The plot of diversity of cluster size shows a tuning effect for the cluster size diversity through parameters $L$ and $p$. In addition, the plot of LA diversity follows a bell-shaped curve centred at the probability of maximum LA diversity.

The inset of these figures shows the ratios $D_{s} / L^{0.98}$ and $D_{f} / L^{1.77}$ as a function of $p$. The diversity of cluster size normalized by $L^{0.98}$ does not collapse for all different values of $L$, indicating that the behaviour of $D_{s}$ is rather complex, so that the exponent of the normalization variable does not follow a simple constant. However, the LA diversity density $D_{f} / L^{1.77}$ shows a fairly good collapse for the different values of $L$. The normalization exponent for the cluster size diversity was obtained from the scaling of $D_{s \max }$ versus $L$ described in the upper inset of figure 7. Similarly, the exponent of the LA diversity was obtained from the scaling of $D_{f \text { max }}$ versus $L$ shown in the lower inset of figure 7 .

In figure 3 we have a log-log plot of the total number of clusters versus the probability of occupation for the various values of $L$. All the plots follow the same behaviour, the variable $N$ increases with $p$, attains a maximum and decreases afterwards. The inset of the figure shows the ratio $N / L^{2}$ as a function of $p$. Similar to the diversity case, the normalization exponent was obtained from the scaling $N_{\max }$ versus $L$ as show in figure 7 . The normalized ratio shows an excellent collapse, except for $p$ close to 1 which is due to finite-size effects.

From percolation and scaling theory we have $\sum_{s} n_{s} \sim\left|p-p_{c}\right|^{2-\alpha}$. The definition of 


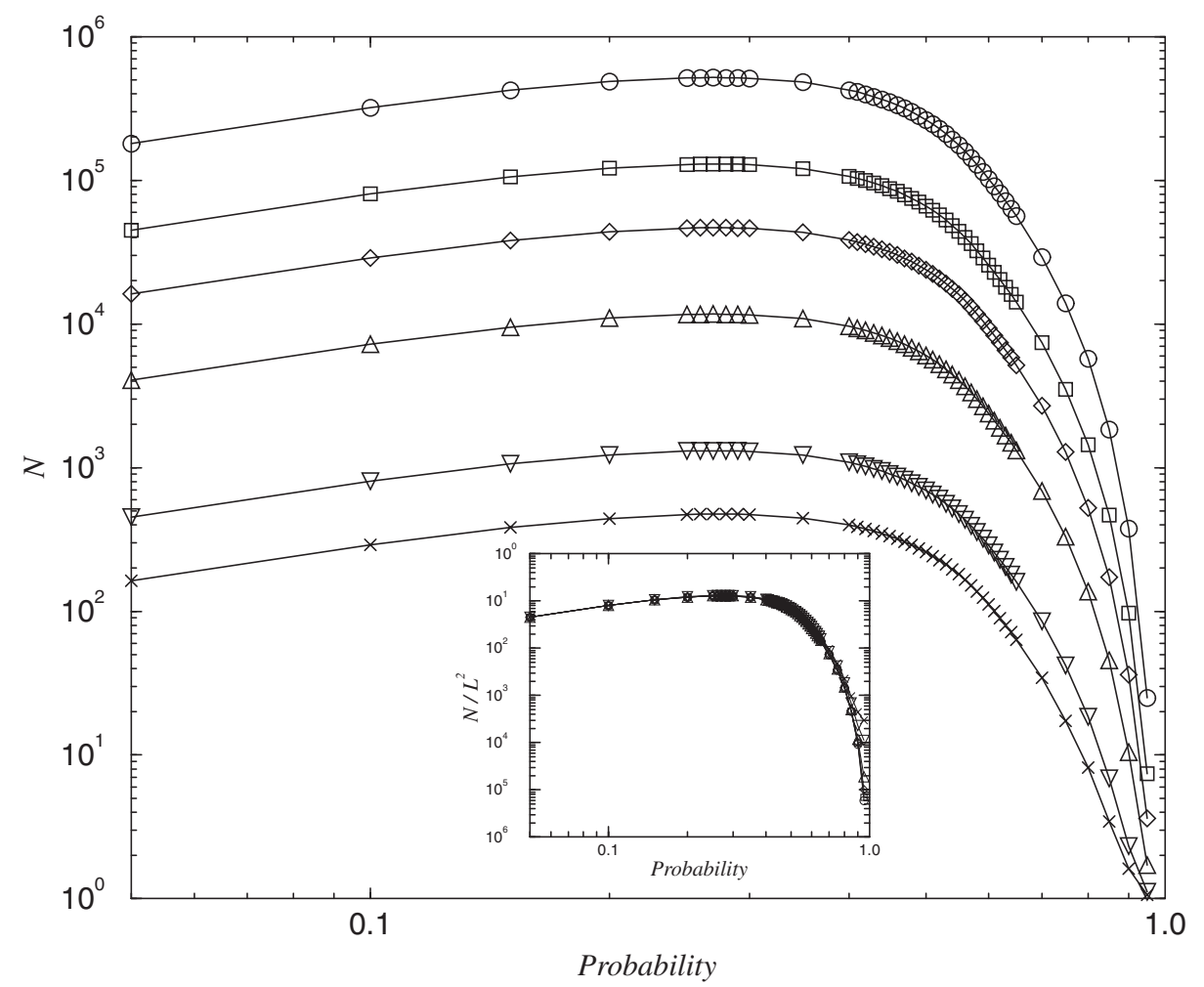

Figure 3. The behaviour of total number of clusters as a function of the probability of occupation for $L=2000(\bigcirc), 1000(\square), 600(\diamond), 300(\triangle), 100(\nabla), 60(\times)$. The inset shows the ratio $N / L^{2}$ against $p$, with the normalization factor all curves collapse.

cluster number is given by $n_{s}=N(s) / L^{d}$, such that $\sum_{s} n_{s} \sim L^{-d}$. Since $L \sim\left|p-p_{c}\right|^{-v}$, we have then $\sum_{s} n_{s} \sim\left|p-p_{c}\right|^{d \nu}$. In this way, we recover the hyperscaling $2-\alpha=d \nu$. This shows that $N$ should in fact scale to $L^{d}$, at the critical point. Furthermore, figure 3 indicates that the scale $N \sim L^{d}$ is valid for all points, not just at the percolation threshold.

In order to determine the critical probability associated with $N_{\max }$ at the thermodynamic limit, that is for $L \rightarrow \infty$, we plot $P_{c}\left(N_{\max }\right)$ versus $1 / L$ as shown in figure $4(a)$. The linear fit gives us $P_{c}\left(N_{\max }\right)=0.27 \pm 0.01$. For the cluster size and LA diversity, the critical probabilities were obtained at $P_{c}\left(D_{s \max }\right)=0.57 \pm 0.02$ and $P_{c}\left(D_{f \max }\right)=0.45 \pm 0.02$ respectively, as shown in figures $4(b),(c)$. Note that $P_{c}\left(D_{s \max }\right)$ is higher than $P_{c}\left(D_{f \max }\right)$, because as $p$ increases less space is left on the lattice for the appearance of different LA. Moreover, different LA are possible with the same cluster size, that is an $n$-cell cluster will generate different LA. To illustrate this situation, figure 5 shows all possible configurations for a fixed LA with size 3 . Here the diversity of fixed LA is equal to 6. On the other hand, if we take the cluster size measurement, we would obtain a diversity equal to 1 . Therefore, the maximum diversity of LA on randomly occupied lattice results from a compromise between a higher value of $p$, generating more clusters with different sizes, and a lower value of $p$, which makes possible the emergence of different LA with the same cluster size.

An important point of interest is the fact that the critical probability of maximum cluster size diversity is obtained at the same point of the percolation threshold ( $\left.p_{c}=0.592746\right)$ [8] from a statistical point of view. We performed more intensive Monte Carlo simulations on 

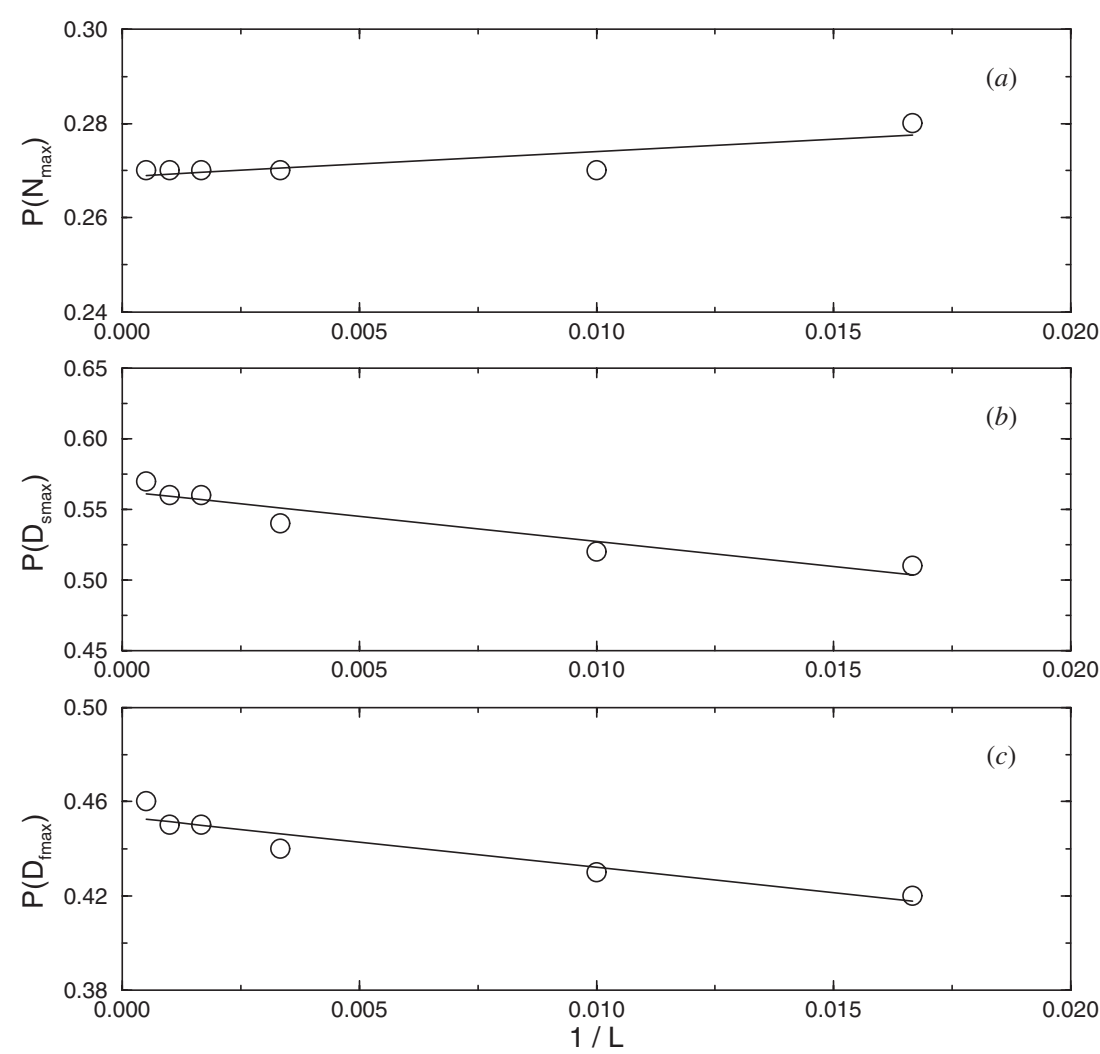

Figure 4. (a) Plot of the linear fit of $P\left(N_{\max }\right)$ as a function of $1 / L$. The straight line has an intercept at $0.27 \pm 0.01$, giving the value for critical probability at the thermodynamic limit. (b) Plot of $P\left(D_{s} \max \right)$ as a function of $1 / L$, yielding the value of $P_{c}\left(D_{s} \max \right)=0.57 \pm 0.02$. (c) Plot of $P\left(D_{f} \max \right)$ versus $1 / L$, yielding the value of $P_{c}\left(D_{f \max }\right)=0.45 \pm 0.02$.
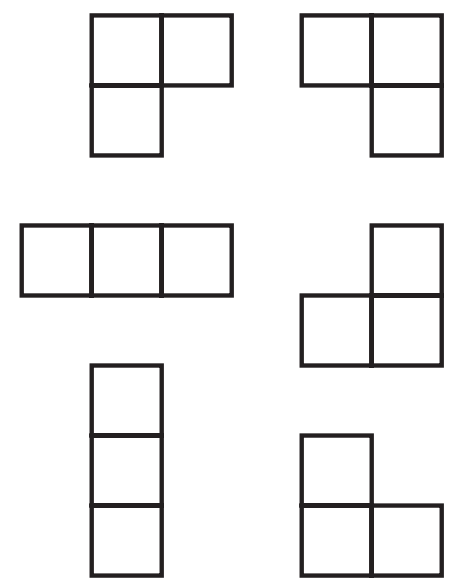

Figure 5. All possible configurations of fixed LA having three cells. In this case, the diversity of LA is equal to 6 while the diversity of cluster size is equal to 1 . Considering free LA the diversity is equal to 2. Note that the maximum diversity of fixed LA for $n$ cells is equal to the total number of possible $n$-cell animals. 


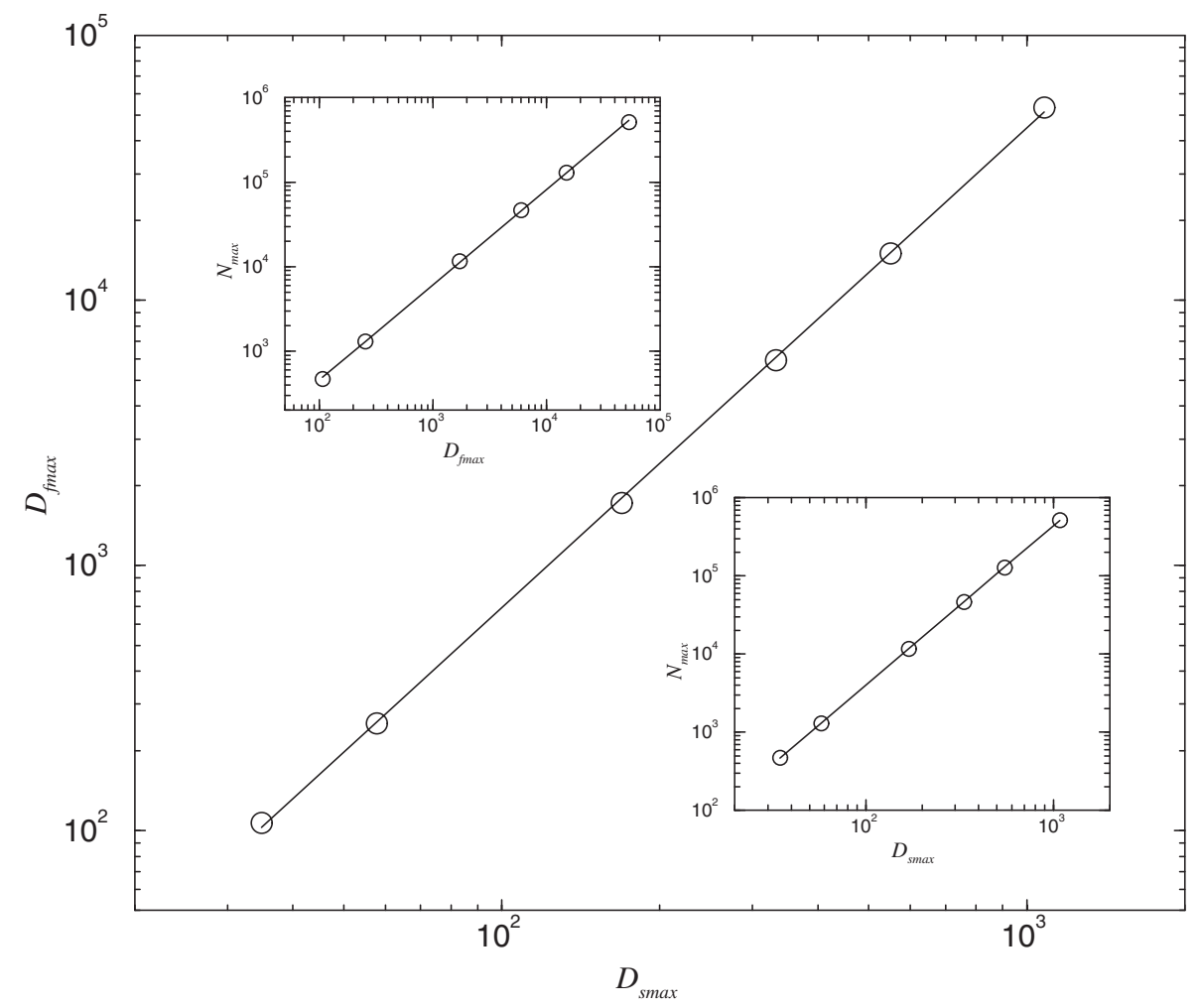

Figure 6. Log-log plot of $D_{f \max }$ versus $D_{s \max }$. The straight line has a slope of value $1.81 \pm 0.05$, giving the non-trivial scaling relation $D_{f \max } \sim D_{s \max }^{1.81 \pm 0.05}$. The upper inset shows the log-log plot of $N_{\max }$ versus $D_{f \max }$ which yields the scaling relation of $N_{\max } \sim D_{f \max }^{1.13 \pm 0.02}$. The lower inset shows the plot of $N_{\max }$ versus $D_{s} \max$ leading to the scaling $N_{\max } \sim D_{s}^{2.01 \pm 0.02}$.

the region near $D_{s \max }$ to verify the validity of this conjecture. Larger lattice sizes were used in these simulations, with sizes varying from $L=2000,4000,6000$ and 8000, and averages taken on 200,100, 50 and 25 experiments. Using finite-size scaling analysis, we obtained the value of $P_{c}\left(D_{s \max }\right)=0.581 \pm 0.005$. The value of $P_{c}\left(D_{s \max }\right)$ will most probably attain the value of $p_{c}$ for the infinite lattice size. For other Euclidean lattice dimensions the conjecture that $P_{c}\left(D_{s \max }\right)=p_{c}$ for $L \rightarrow \infty$ is also shown to be valid [25]. An analytical approach for the derivation of this probability is still lacking. This solution will possibly bring important contributions to the percolation problem. However, such approach may face unsolved difficulties as for example the solution for the exact enumeration of the LA.

\section{Scaling relations}

The scaling behaviour of the maximum number of fragments and the maximum cluster size diversity follows $N_{\max } \sim D_{s \max }^{2.01 \pm 0.02}$. This result is shown to be the same as the experiments on different fragmentation and aggregation processes [19,22-24,29,30]. Furthermore, the scaling $N_{\max } \sim D_{f \max }^{1.13 \pm 0.02}$ presents a lower exponent, due to the fact that $D_{s} \leqslant D_{f}$ for the same lattice size $L$. Since LA are a more refined method to distinguish the clusters in comparison to cluster size. These exponents are of interest since they relate the diversity (complexity) of the system 


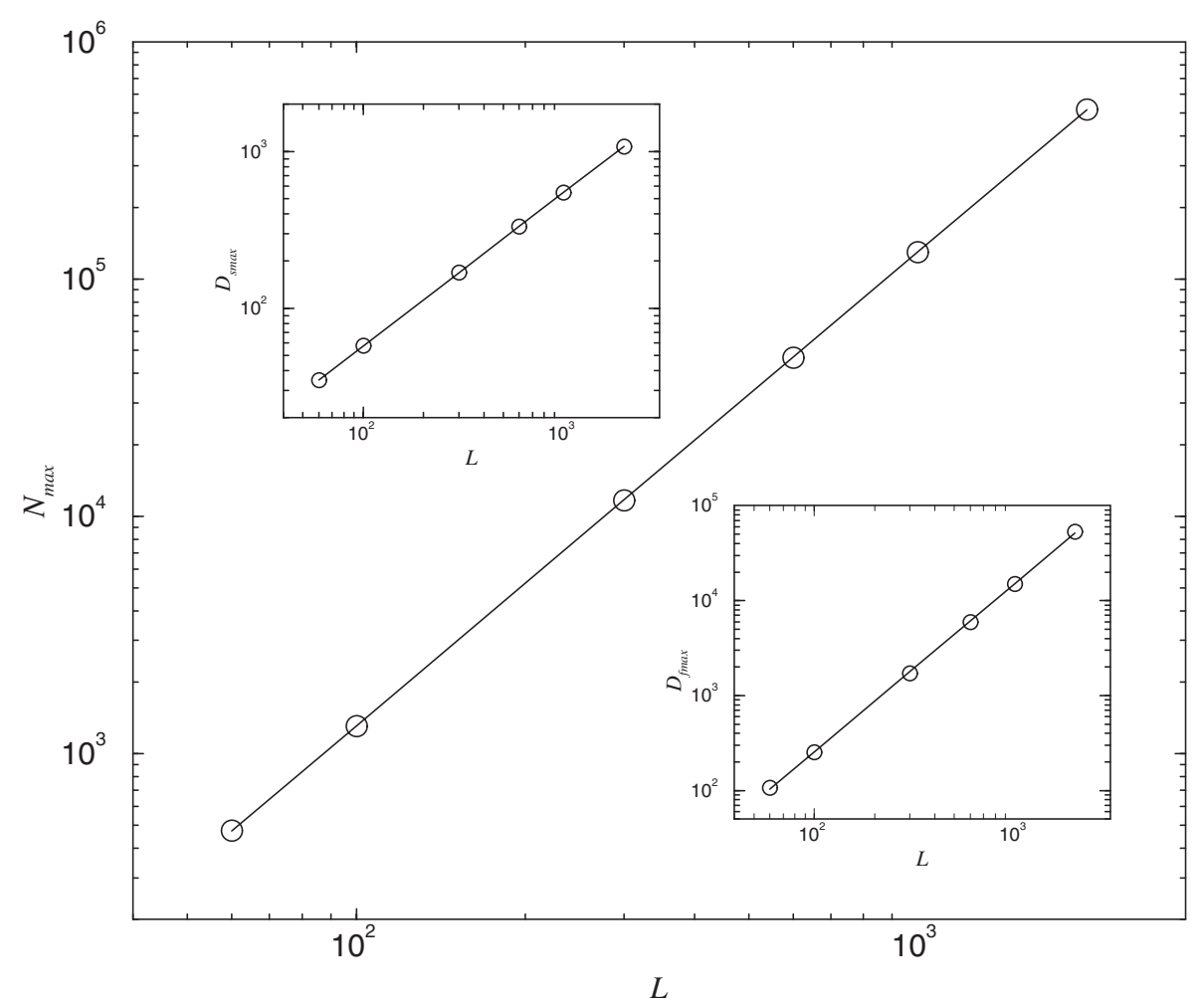

Figure 7. $\log -\log$ plot of $N_{\max }$ versus $L$. The straight line has a slope of value $2.00 \pm 0.02$, yielding the scaling relation $N_{\max } \sim L^{2.00 \pm 0.02}$. The upper inset shows the log-log plot of $D_{s} \max$ versus $L$ which yields the scaling relation of $D_{s \max } \sim L^{0.98 \pm 0.03}$. The lower inset shows the plot of $D_{f \text { max }}$ versus $L$ leading to the scaling $D_{f \max } \sim L^{1.77 \pm 0.03}$.

with the total number of fragments (population of clusters). The plots of these scalings are shown in the upper and lower insets of figure 6. For the cluster size diversity, this exponent is very robust $[18,19]$, accordingly one might expect the same robustness for the LA diversity. In figure 6 , we also have the scaling relation of $D_{f \max }$ versus $D_{s \max }$, with a slope value of $1.81 \pm 0.05$. This scaling shows how the maximum diversity of LA increases in relation to the maximum diversity of cluster size for different lattice size.

The scaling between the maximum number of clusters and the lattice size of the system is given by $N_{\max } \sim L^{2.00 \pm 0.02}$. In the cases of maximum cluster size and LA diversity such scaling follows $D_{f s \max } \sim L^{0.98 \pm 0.03}$ and $D_{f \max } \sim L^{1.77 \pm 0.03}$, respectively. These scaling relations can be seen in figure 7 . The difference between the cluster size and LA diversity exponents is again due to the fact that $D_{s} \leqslant D_{f}$.

The scaling behaviour analysed so far relates specific points, i.e. the points where the measurements of interest attain a maximum. This is of interest, because the point of maximum cluster size diversity is found to be statistically the same as of the percolation threshold. However, the study of the behaviour of the variables according to the probability of occupation, $p$, is of importance. So that a better understanding of the underline physical process of the system, either as a fragmentation/aggregation process or as a geometrical phase transition phenomena (percolation) can be acomplished. Accordingly, the scaling relation of $N, D_{s}$ and $D_{f}$ versus $L$ can be obtained for each value of $p$. It is also possible to determine the effective 

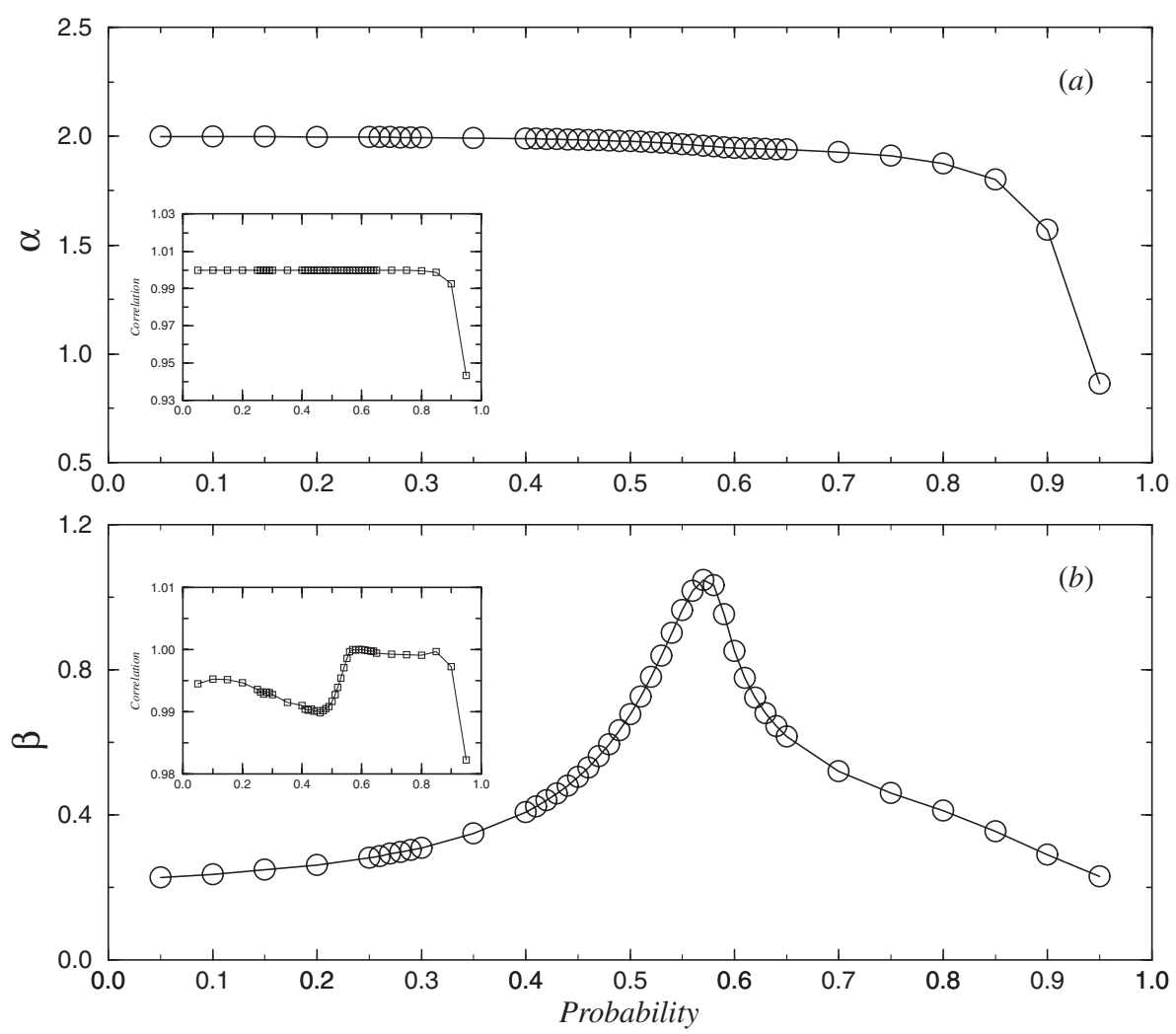

Figure 8. The effective exponents (a) $\alpha$ versus $p$ obtained from the scaling relation $N(p) \sim L^{\alpha(p)}$. The inset of this figure show the correlation coefficient of this scaling. For most values of $p$ this number is equal to 1 . However, for the values of $p$ near 1, we have a decay on the value of $\alpha$ and of the correlation coefficient, which is due to finite-size effects. (b) $\beta$ versus $p$ from the scaling $D_{s}(p) \sim L^{\beta(p)}$. The inset shows the correlation coefficient for this plot.

exponents $\alpha, \beta$ and $\delta$ as a function of $p$. The scalings follow as:

$$
\begin{aligned}
& N(p) \sim L^{\alpha(p)} \\
& D_{s}(p) \sim L^{\beta(p)} \\
& D_{f}(p) \sim L^{\delta(p)}
\end{aligned}
$$

and hence

$$
D_{f}(p) \sim D_{s}(p)^{\lambda(p)}
$$

where $\lambda(p)=\delta(p) / \beta(p)$.

The behaviour of the effective exponent $\alpha$ versus $p$ is shown in figure 8(a). The exponent $\alpha$ maintains constant around the value 2 for almost all values of $p$. As $p$ increases the system attains a configuration characterized by a main connected cluster with few small clusters so that the $\alpha$ exponent decreases. No scaling is observed as the value of $p$ gets very close to 1 , since just one connected cluster emerges on the lattice. The inset of this figure shows the correlation coefficient for these scalings. For most of the values of $p$ the correlation is practically equal to 1 . This indicates that the power-law dependence follows a reasonable good fit, except for $p$ close to 1 , due to finite-size effects. According to section $4, \alpha(p)$ is found to be equal to the dimension of the lattice, irrespective of $p$, see [25]. The analyses of these effective exponents 

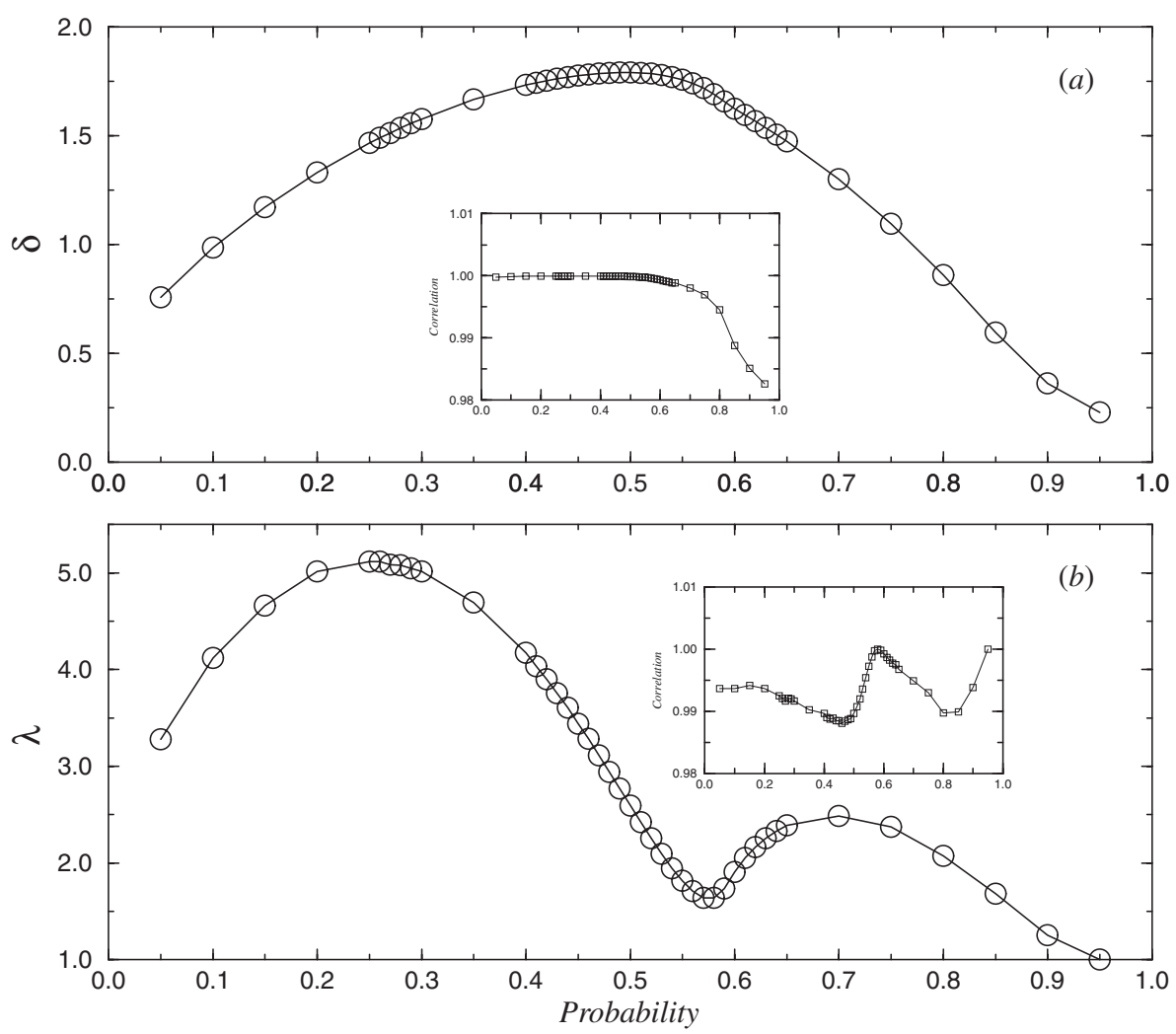

Figure 9. The effective exponents $(a) \delta$ versus $p$ obtained from the scaling relation $D_{f}(p) \sim L^{\delta(p)}$. The inset of this figure show the correlation coefficient of this scaling. $(b) \lambda$ versus $p$ from the scaling $D_{f}(p) \sim D_{s}(p)^{\lambda(p)}$. The inset shows the correlation coefficient for this plot.

are of interest because they give a good indication on how those exponents should behave when the size of the system goes to infinity.

The behaviour of $\beta$ differs significantly from $\alpha$, since $\beta$ depends highly on the value of $p$ and attains a maximum at the critical point, see figure $8(b)$. This indicates that the rate of increase in diversity for different values of $L$ is higher in the region of the maximum. The behaviour of the correlation coefficient is shown in the inset of this figure. In the region of $p \leqslant p_{c}$, the correlation coefficient decreases, attains a minimum and increases until 1 at $p_{c}$. Although, the correlation coefficients are close to 1 , this implies that as the system size goes to infinity the value of $\beta$ in this region will need some corrections. The $\beta$ curve will probably present a sharper increase. For $p \geqslant p_{c}$ the correlation coefficient is very close to 1 , showing that those exponents are more accurate. Here we find a slight concave curve implying the value of $\beta$ must sharply decline for $p \geqslant p_{c}$. In addition, for $p$ close to 1 the correlation falls in the same way as $\alpha$, this is also due to finite-size effects. By calculating $\beta$ using small values of $L$ and progressively recalculate it with larger $L$, we conclude that the overall form of the curve as $L \rightarrow \infty$ will present an accentuate peak at $p_{c}$.

A similar scaling relation is obtained for the LA diversity following equation (6) and shown in figure $9(a)$. However, the curve of the exponent $\delta$ as a function of $p$ has a different shape compared with $\beta$, which is more peaked at the maximum region. The inset shows that the correlation coefficients curve have a shape similar to the one obtained with the exponent 

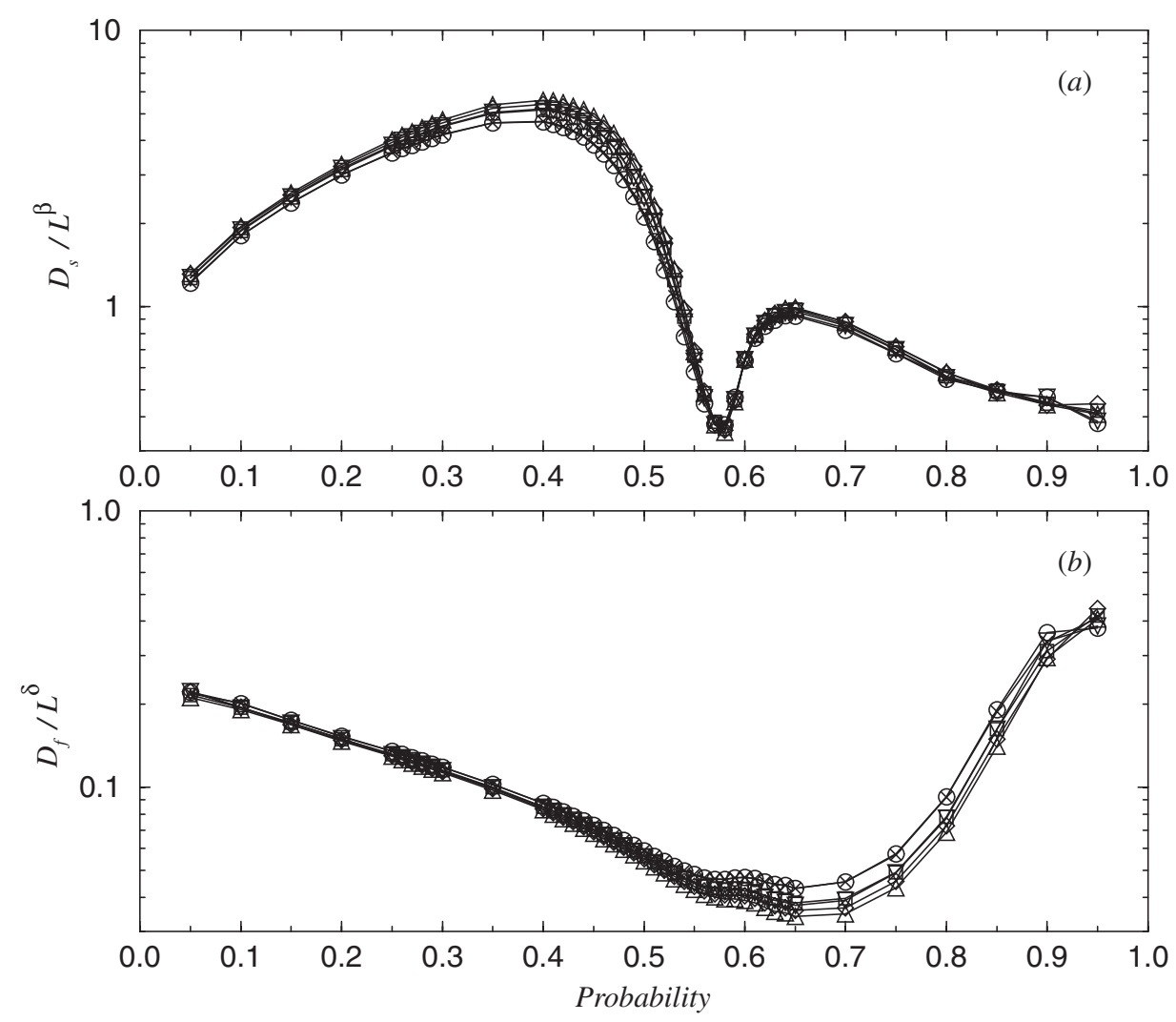

Figure 10. The collapsing curves of (a) the cluster size diversity normalized by $L^{\beta}$ as a function of $p$, for several lattice sizes. (b) The same as figure (a) but for the LA diversity with a rescaled normalization of $L^{\delta}$.

$\alpha$. These coefficients are very close to 1 , which indicates that the power-law scaling provides a good fit not just at the critical point.

In figure $9(b)$ we have the plot of the effective exponent $\lambda$ from the scaling relation described by equation (7). It is interesting to observe that $\lambda$ attains its maximum value at the same point where the system attains a configuration with the maximum amount of clusters, $P_{c}\left(N_{\max }\right)$. This probability has a relatively low value which leads to the appearance of different LA with the same cluster size. Moreover, as $p$ equals the maximum cluster size diversity, $\lambda$ attains a local minimum, because the diversity of cluster size, in comparison to LA diversity, is limited by the lower bound $D_{s} \leqslant D_{f}$. At $P_{c}\left(D_{s \max }\right), D_{s}$ attains the nearest value to $D_{f}$, except on the two uninteresting states where $p$ is either close to 0 or 1 . In these two extreme points $\lambda$ attains a value equal to 1 , since $D_{s}$ is equal to $D_{f}$. In the inset of the figure, we show the correlation coefficient for these exponents. Note that, for $p \leqslant p_{c}$ the curve is very similar to the correlation coefficient obtained for $\beta$. However, for $p \geqslant p_{c}, \lambda$ decreases, attains a minimum and increases until $p=1$, reaching the value 1 since at this point $D_{s}=D_{f}$.

In the inset of figure 1 , we have shown that diversity density $D_{s} / L^{0.98}$ does not collapse the curves for all different $L$. Hence, it is necessary to include a scaling factor having the exponent as a function of $p$. We obtain the desired collapsing curves, using the effective 


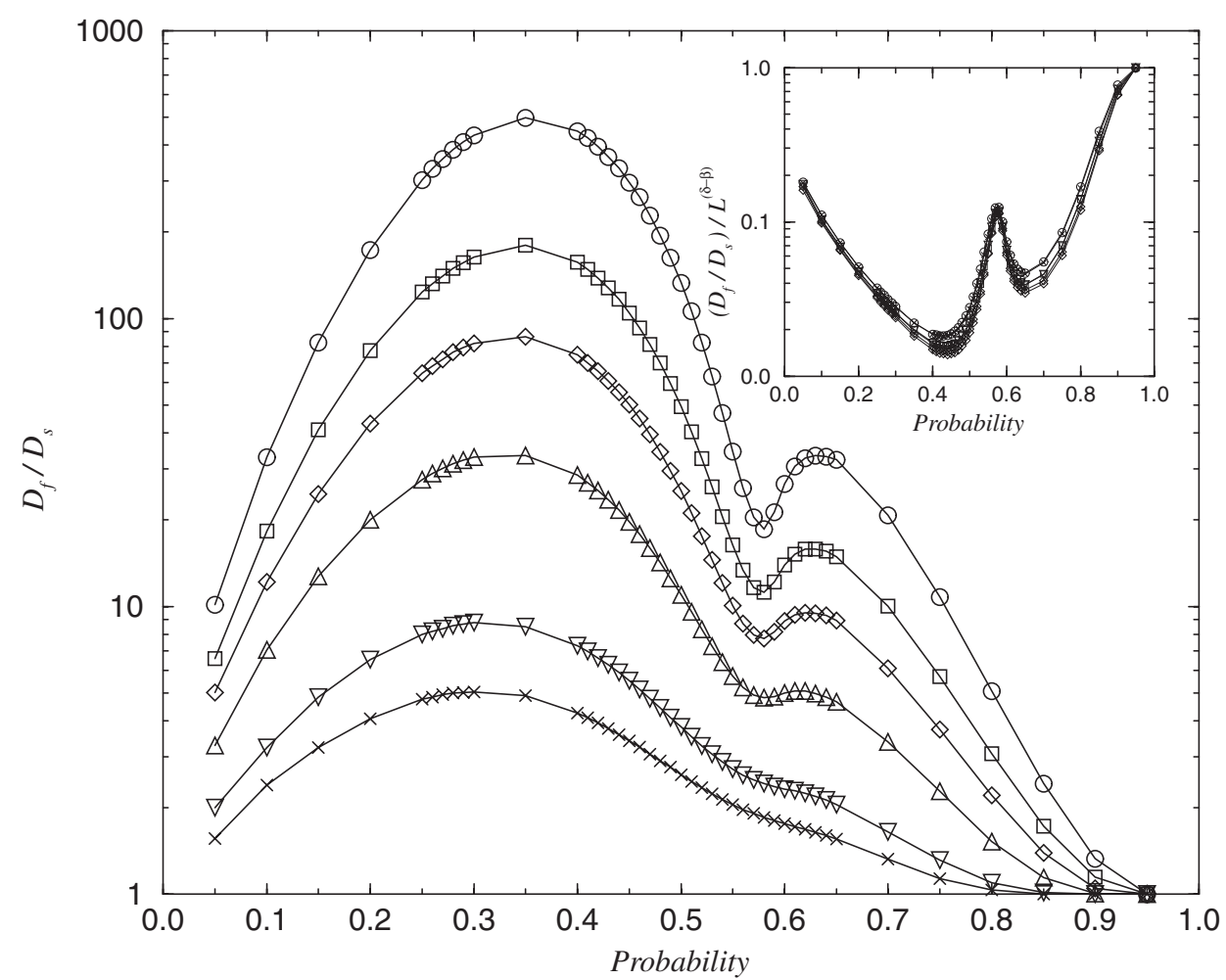

Figure 11. The ratio $D_{f} / D_{s}$ as a function of $p$ for $L=2000(\bigcirc), 1000(\square), 600(\diamond), 300(\triangle)$, $100(\nabla), 60(\times)$. The inset shows this ratio normalized by $L^{(\delta-\beta)}$ so that all curves collapse.

exponent $\beta$. Figure 10(a) shows a plot of this curve. This rescaled curve is characterized by two maxima and an interesting minimum point at $p_{c}$. The same kind of rescaling can be applied for the LA diversity, even though the normalization by $L^{1.77}$ shows a reasonable good collapse. Figure $10(b)$ shows the resulting curve, rescaled by the factor $L^{\delta}$. The fact that, at a fixed probability, $D_{s}$ scale with $\beta$ and $D_{f}$ scale with $\delta$, implies that the average diversity densities $D_{s} / L^{d}$ and $D_{f} / L^{d}$ decay as $L^{(\beta-d)}$ and $L^{(\delta-d)}$, respectively. Therefore, $D_{s}$ and $D_{f}$ measured on a finite system must be rescaled according to the dimension and size of the system, to correctly correspond to a large system.

The ratio $D_{f} / D_{s}$ as a function of the probability of occupation for different lattice sizes is shown on the figure 11. Taking the dependence of the size of the system $L$ into consideration, so that all the curves collapse, a normalization of the following form is necessary: $\left(D_{f} / D_{s}\right) / L^{(\delta-\beta)}$. This collapsing curve is shown in the inset of the figure.

The variability of the system is defined as the ratio diversity divided by the total number of clusters or the population of the system for each value of $p$. In figures 12 and 13 we have the plots of these ratios for the various values of $p$. For a low probability of occupation the ratio is close to zero, since $N$ has a high value and both $D_{s}$ and $D_{f}$ have a very low value. However, for $p$ near 1 the ratio is equal to one, because of the single connected cluster state. The total number of clusters scales with the system size as $N(p) \sim L^{d}$, in which $d$ is equal to the dimension of the system. Using the effectives exponents found through equations (5) and (6), we have that the normalizations should follow $\left(D_{s} / N\right) / L^{(\beta-d)}$ and $\left(D_{f} / N\right) / L^{(\delta-d)}$, respectively. The inset of these figures show the collapse of curves for the different values of 


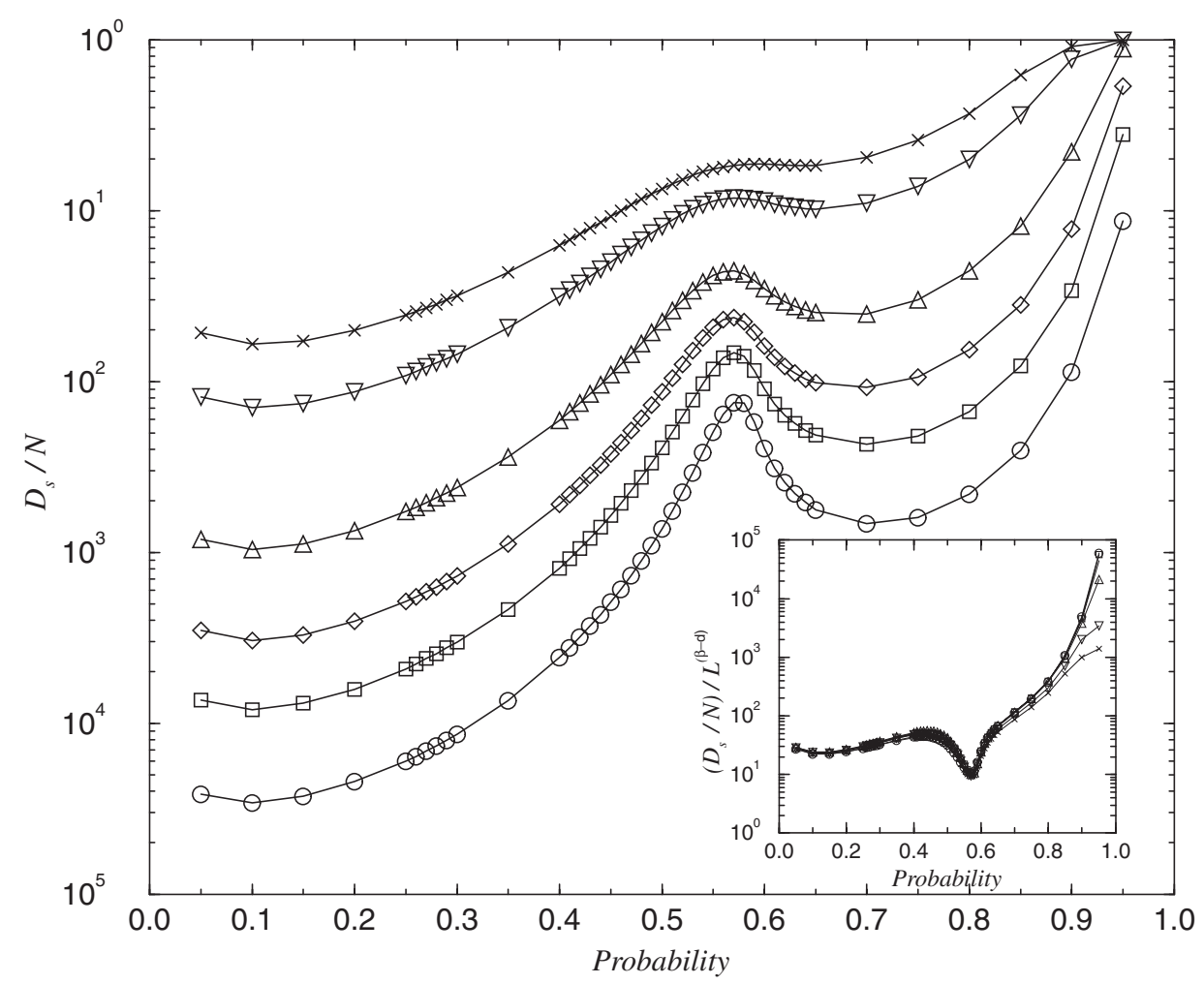

Figure 12. The variability ratio $D_{s} / N$ as a function of $p$. The inset shows this ratio normalized by $L^{(\beta-d)}$ so that all curves collapse.

$L$ as a function of $p$. These ratios are of interest because they show the relation between the diversity (complexity) of the system and the total number of clusters for all values of $p$.

\section{Discussions and conclusions}

In this paper, we investigated the different cluster configurations generated on a randomly occupied square lattices. The process studied is one of the simplest models of disordered systems, though different from fragmentation and aggregation processes, since there are no dynamics involved in these simulations. In most of the studies of percolation and random disordered systems, the statistics of the average number of clusters, $n_{s}$, and the cluster size distribution have been thoroughly analysed. In various models, those variables are reasonably well understood. In contrast, the statistics of cluster size diversity is a variable not as greatly investigated. Nevertheless, some studies on fragmentation [18, 19,22], cellular automata [20,21], random walks [30], and more recently on randomly occupied lattices [23-25] have been reported. Diversity of cluster size has also been used as a measurement of the structural complexity [22, 24, 29].

Here, we presented an analysis on the LA diversity in contrast with cluster size diversity. We described the behaviour of cluster size and fixed LA diversity on randomly occupied square lattice. A critical probability associated with the maximum of LA diversity was introduced, in comparison with the critical probability of the maximum cluster size diversity and the total 


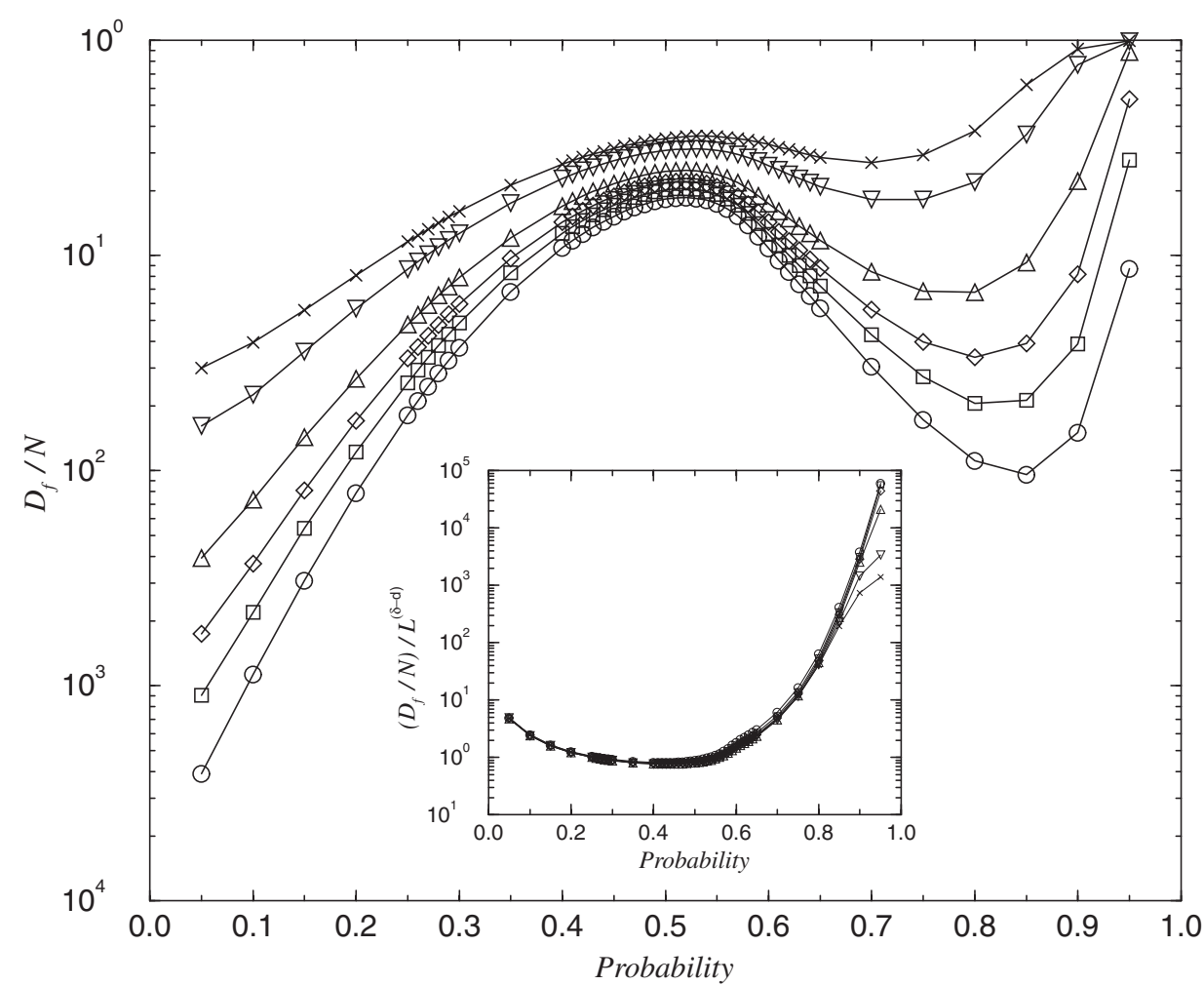

Figure 13. Similar to the plot in figure 12 but for the LA diversity. The inset shows the ratio normalized by $L^{(\delta-d)}$.

number of clusters. Several scaling relations between the measured variables, the lattice size and the probability of occupation were derived. We described the algorithm used to identify and count the different LA. This algorithm, with some modifications, is also able to identify free LA, so that similar expressions and curves could be obtained. However, for this particular model, they would yield results analogous to the fixed LA.

In order to determine a more accurate number for the critical probability of maximum cluster size diversity, we have performed intensive simulations with lattice sizes of up to 8000 . The result indicates that $P_{c}\left(D_{s \max }\right)$ has statistically the same value as the percolation threshold, $p_{c}$. Experiments with other lattice dimensionalities [25] also support this conjecture. If we take the fact that the correlation length diverges at $p_{c}$, and that all length scales are present at this probability. It is reasonable that maximum cluster size diversity also occurs at the percolation threshold.

Critical exponents can also be derived from those measurements and presently we are working on this direction. We believe that the study of diversity can improve our knowledge and understanding of this and other models which generate a cluster distribution and in addition, in a more general sense, to the understanding of the geometrical phase transition in the percolation problem. 


\section{Acknowledgment}

I J Tsang and I R Tsang would like to acknowledge the Brazilian agency CAPES for financial support.

\section{References}

[1] Family F 1983 J. Phys. A: Math. Gen. 16 L97

[2] Guttmann A J 1982 J. Phys. A: Math. Gen. 151987

[3] Dhar D, Phani M K and Barma M 1982 J. Phys. A: Math. Gen. 15 L279

[4] Conway A 1995 J. Phys. A: Math. Gen. 28 L125

[5] Domb C 1976 J. Phys. A: Math. Gen. 9 L141

[6] Redner S and Coniglio A 1982 J. Phys. A: Math. Gen. 15 L273

[7] Conway A R and Guttmann A J 1995 J. Phys. A: Math. Gen. 28891

[8] Stauffer D and Aharony A 1994 Introduction to Percolation Theory (London: Taylor and Francis)

[9] Sahimi M and Jerauld G R 1984 J. Phys. A: Math. Gen. 17 L165

[10] Alexander S, Grest G S, Nakanishi H and Witten T A Jr 1984 J. Phys. A: Math. Gen. 17 L185

[11] Shahverdian A Y 1997 Fractals 5199

[12] Golomb S W 1994 Polyominoes: Puzzles, Patterns, Problems, and Packings (Princeton: Princeton University Press)

[13] Thompson D M 1971 On Growth and Form (Cambridge: Cambridge University Press)

[14] Raup D M, Gould S J, Schopf T J M and Simberloff D S 1973 J. Geol. 81525

[15] Kauffman S A 1993 The Origins of Order (Oxford: Oxford University Press)

[16] Wolfram S 1994 Cellular Automata and Complexity: Collected Papers (Reading, MA: Addison-Wesley)

[17] Mandelbrot B B 1983 The Fractal Geometry of Nature (New York: Freeman)

[18] Gomes M A F and Vasconcelos G L 1989 J. Phys. A: Math. Gen. 22 L757

[19] Coutinho K, Gomes M A F and Adhikari S K 1992 Europhys. Lett. 18119

[20] Garcia J B C, Gomes M A F, Jyh T I, Ren T I and Sales T R M 1993 Phys. Rev. E 483345

[21] Sales T R M, Garcia J B C, Jyh T I, Ren T I and Gomes M A F 1993 Physica A 197604

[22] Gomes M A F, Souza F A O and Adhikari S K 1995 J. Phys. A: Math. Gen. 28 L613

[23] Tsang I R and Tsang I J 1997 J. Phys. A: Math. Gen. 30 L239

[24] Tsang I J and Tsang I R 1999 Unifying Themes in Complex Systems: Proc. 1st Necsi Int. Conf. on Complex Systems ed Y Bar-Yam (Reading, MA: Perseus Book) also available at InterJournal webpage http://www.interjournal.org/

[25] Tsang I R and Tsang I J 1999 Phys. Rev. E 602684

[26] Hoshen J and Kopelman R 1976 Phys. Rev. B 143438

[27] Hoshen J, Berry M W and Minser K S 1997 Phys. Rev. E 561455

[28] Tsang I J and Tsang I R 1999 unpublished

[29] Garcia J B C, Gomes M A F, Jyh T I, Ren T I and Sales T R M 1999 The Evolution of Complexity ed F Heylighen, J Bollen and A Riegler (Dordrecht: Kluwer)

[30] Coutinho K R, Coutinho-Filho M D, Gomes M A F and Nemirovsky A M 1994 Phys. Rev. Lett. 7237 